Open Access Full Text Article

\title{
Increased Energy Expenditure and Energy Loss Through Feces Contribute to the Long-Term Outcome of Roux-en-Y Gastric Bypass in a Diet-Induced Obese Mouse Model
}

This article was published in the following Dove Press journal:

Diabetes, Metabolic Syndrome and Obesity: Targets and Therapy

\section{Kai Chen* \\ Boen Xiao* \\ Zhe Zhou \\ Weihui Peng \\ Wei Liu}

Department of Biliopancreatic Surgery and Metabolic Surgery, Second Xiangya Hospital, Central South University, Changsha, People's Republic of China

*These authors contributed equally to this work
Correspondence: Wei Liu Email liuweixy@csu.edu.cn
Background: Roux-en-Y gastric bypass (RYGB) has been proved to be more effective than other bariatric procedures in the long term on body-weight loss and remission of diabetes. However, the mechanism remains poorly understood. Long-term changes in energy metabolism after RYGB have rarely been reported.

Objective: To investigate the long-term effects of RYGB on energy metabolism on a dietinduced obesity (DIO) mouse model.

Methods: DIO mice fed a high-fat diet were assigned to two groups: RYGB ( $n=8)$ and sham $(n=7)$, followed by high-fat diet feeding until 12 weeks after surgery. Body weight and food intake were recorded weekly. Measurement of body composition and energy metabolism by metabolic chamber were conducted at weeks 4, 8, and 12 after surgery. Fecal energy measurement, intraperitoneal glucose-tolerance tests, and insulin-tolerance tests were conducted at postoperative week 12. Results: Food intake was reduced in the RYGB group within the first 3 weeks after surgery and increased to the same as the sham group from postoperative week 4 . At 12 weeks after surgery, body weight had reduced by $36 \% \pm 3.2 \%$ in the RYGB group compared to a $16 \% \pm 2 \%$ body-weight gain in the sham group, while fat mass had reduced significantly in the RYGB group compared to the sham group $(9.2 \% \pm 1.5 \%$ versus $30.1 \% \pm 0.7 \%)$. Energy expenditure was significantly higher at postoperative week 8 in the RYGB group than the sham group. In comparison with the sham group, the respiratory exchange ratio was unchanged, decreased, and increased in the RYGB group at postoperative weeks 4,8 , and 12 , respectively. Fecal energy measurement showed that feces from mice in the RYGB group contained higher energy levels than the sham group. Glucose metabolism had significantly improved in the RYGB group, in contrast to the sham group, demonstrated by intraperitoneal glucose tolerance tests (AUC 1,502 \pm 104 versus 2,277 \pm 198 , respectively) and insulin tolerance tests (AUC $524 \pm 50$ versus $838 \pm 63$, respectively).

Conclusion: Increased energy expenditure and energy loss through feces contribute to longterm body-weight control after RYGB. Enhanced glucose utilization might play a role in long-term improvement in glucose metabolism.

Keywords: Roux-en-Y gastric bypass, mouse model, glucose metabolism, energy expenditure, respiratory exchange ratio

\section{Introduction}

Bariatric surgery has been reported to be effective not only for body-weight loss but also for improvement in obesity-related comorbidities, such as type 2 diabetes mellitus (T2DM). ${ }^{1,2}$ Roux-en-Y gastric bypass (RYGB) and sleeve gastrectomy are 
superior to medical treatment for T2DM, according to a 5-year prospective randomized clinical trail, which led to the consensus that bariatric surgery should be listed in the treatment algorithm of T2DM. ${ }^{3,4}$ Moreover, RYGB shows better long-term effects than vertical sleeve gastrectomy, which raises interest in physiological changes in the long term after RYGB. ${ }^{3}$ However, the mechanism underlying the remission of T2DM after RYGB remains poorly understood. Different animal models of bariatric surgery have been used, ${ }^{5-7}$ but creating a mouse model of RYGB is a technical challenge. Long-term metabolic effect of RYGB in mouse models have rarely been reported. Based on our experience with a mouse model of duodenojejunal bypass ${ }^{8}$ and other reports, ${ }^{9,10}$ we refined the surgical technique and successfully established an effective RYGB mouse model with no mortality. In this report, the long-term effect of RYGB is presented, with analysis of energy metabolism.

\section{Methods}

\section{Animals and Diet}

Male C56BL/6J mice aged 7 weeks (Hunan SJA Laboratory Animal) were housed in groups under 12-hour light/dark cycles (lights on at $7 \mathrm{am}$ ) and a room temperature of $21^{\circ} \mathrm{C}-23^{\circ} \mathrm{C}$, and provided food and water ad libitum before surgery. Upon arrival at the Second Xiangya Hospital of Center South University Animal Research Center, mice were introduced to a high-fat diet $(60 \% \mathrm{kcal} \%$; Research Diets, D12492) for 12 weeks until they reached a body weight of 40-50 g (we identified obesity as body weight $>25 \%$ compared to age-matched chow-fed counterpart ${ }^{11}$ ). Mice were assigned to two groups according to body-weight match: RYGB ( $=8)$ and sham $(n=7)$. Mice were feed with Ensure as liquid diet at 24 hours before surgery. The body weight of mice was not significantly different between the groups before surgery. Under the Guide for Care and Use of Laboratory Animals issued by the Ministry of Science and Technology of China in 2006, the Institutional Animal Care and Use Committee of Second Xiangya Hospital of Central South University approved all experiments.

\section{Anesthetization}

General anesthesia was induced in the induction chamber with $5 \%$ isoflurane $(0.4 \mathrm{~L} / \mathrm{min})$ and $\mathrm{O}_{2}$ (dioxygen; $\left.0.4 \mathrm{~L} / \mathrm{min}\right)$. After loss of consciousness, mice were transferred to the operating table and continuously anesthetized with $2 \%$ isoflurane $(0.4$ $\mathrm{L} / \mathrm{min}$ ) and $\mathrm{O}_{2}$ (dioxygen; $0.4 \mathrm{~L} / \mathrm{min}$ ) through a nozzle of the anesthesia system. Mice were placed on heating pads in dorsal recumbency. Abdominal skin was molted by depilatory cream and disinfected with povidone-iodine swabs. The entire body was covered with sterile surgical drape. During surgery, body core temperature was maintained within $30^{\circ} \mathrm{C}-32^{\circ} \mathrm{C}$. Respiration rate and toe-pinch tests were used to confirm the depth of anesthesia.

\section{Roux-en-Y Gastric Bypass}

Before this study, we performed RYGB on 17 diet-induced obesity (DIO) mice, but postoperative mortality was very high. Then, based on our previous experience with duodenojejunal bypass ${ }^{8}$ and the modified RYGB model reported by Yin et al, ${ }^{9}$ we made technical improvements to minimize surgical complications. A $2 \mathrm{~cm}-$ long midline incision was made right below the xiphoid process and then two stay stitches placed on each side of the incision for retraction to expose the abdomen. The mesocolon was exposed and the ligament of Treitz identified by moving the small bowel with a moistened cotton bud, then the jejunum was ligated with an $8 / 0$ suture at $4 \mathrm{~cm}$ distally to the ligament of Treitz, where normally there is a nonvascular gap on the mesentery. The jejunum was transected at the right proximally to the ligation, and as a result the proximal part of jejunum was the biliopancreatic (BP) limb (Figure 1A).

The designated alimentary (AL) limb measured $4 \mathrm{~cm}$ from the point of ligation on the distal part of the jejunum, and a $2 \mathrm{~mm}$ longitudinal incision was made at the end of the AL limb in preparation for subsequent anastomosis. Then, end-to-side jejunojejunostomy was performed between the BP limb and the AL limb in an interrupted fashion with 11/0 suture (Figure 1B). Small, saline-soaked gauze was placed around the liver, stomach, and spleen to expose the epigastric area, and ligaments between the liver and distal part of the esophagus were divided to expose the esophagogastric junction (EGJ). The EGJ was ligated with $8 / 0$ suture and the suture then stitched to a saline-soaked gauze ball as a retraction to expose the distal part of the esophagus. A $2 \mathrm{~mm}$-long incision in the esophagus on the right of and superior to the suture ligation was performed with scissors (Figure 1C). The proximal end of the AL limb was placed near the EGJ and a $2 \mathrm{~mm}-$ long incision was made, then side-to-side anastomosis was performed between the distal part of the esophagus and the AL limb with running 11/0 suture (Figure 1D). The abdominal incision was closed with $4 / 0$ absorbable suture after realignment of the small bowel, followed by subcutaneous 


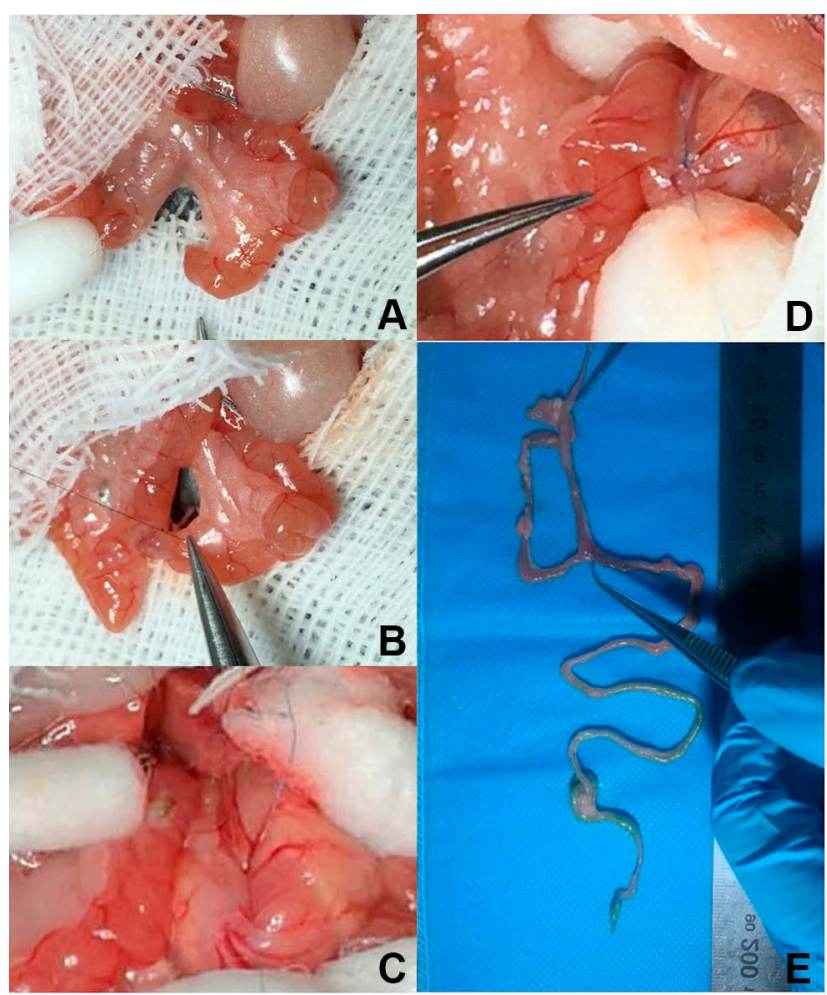

Figure I Surgical techniques of RYGB. (A) Jejunum transected proximally to the ligation; (B) end-to-side jejunojejunostomy between BP limb and AL limb; (C) $2 \mathrm{~mm}$-long incisions on $\mathrm{AL}$ limb and distal part of esophagus just superior to EGJ; (D) side-to-side anastomosis between distal part of esophagus and AL limb; (E) entire alimentary tract showed completely excluded stomach and patent anastomosis after RYGB.

injection of $1 \mathrm{~mL}$ saline $(0.9 \%)$ for fluid compensation and $1 \mathrm{mg} / \mathrm{kg}$ buprenorphine.

\section{Surgical Procedure in Sham Group}

The Sham procedure consisted of laparotomy, jejunal transection $4 \mathrm{~cm}$ distally to the ligament of Treitz, and reanastomosis in situ. Sham-group mice had the same operating time and received the same perioperative care.

\section{Postoperative Care}

Mice were placed on an electric blanket to regain consciousness and continuously monitored until normal behavior (walking and standing without falling), before being transferred to a cage and given water and liquid al libitum. The incubator temperature was set at $30^{\circ} \mathrm{C}$. A solid highfat diet was resumed once defecation had been observed. Because surgical stress can inhibit mouse grooming habits and lead to hair wetting, we used dry swabs to groom them twice a day. Mice produced loose and shapeless stools for about 2 weeks after RYGB, which can lead to rectocele. We nursed anuses with normal saline daily.

\section{Body Weight and Composition}

Body weight was measured every Thursday between 10 and 12 am throughout the duration of the study. Body composition - fat mass, lean mass, and fluid mass - was measured with Dexa (GE Medical Systems, Madison, WI) at 0, 4, 8, and 12 weeks after surgery. The adiposity index was calculated by dividing fat mass by lean mass.

\section{Food Intake}

Food intake was measured weekly by housing the mice individually for 24 hours on an elevated grid floor without bedding. Intake was calculated by subtracting the amount of food remaining plus spillage found under the grid floor (distinguishable by the blue high-fat crumbs) from the previous day's measurement.

\section{Fecal Energy Analysis}

Feces were collected over 24 hours in postoperative week 12. Feces were weighted and kept in a $-80^{\circ} \mathrm{C}$ refrigerator. Fecal gross energy was measured using a ballistic bomb calorimeter (C6000; IKA, Staufen, Germany) after feces had been dried in an oven. Results are presented as thermal energy per gram of feces.

\section{Metabolic Chambers}

Oxygen consumption and carbon dioxide output were measured in metabolic chambers at 4, 8, and 12 weeks after surgery. Mice were transferred to a Comprehensive Laboratory Animal Monitoring System (Columbus Instruments, OH, USA) under a 12-hour/12-hour day/night cycle (lights on at $7 \mathrm{am}$ ). Mice were adapted to the cage for 24 hours before each trial. Data were analyzed with Oxymax software (Columbus Instruments). Equations used were respiratory exchange ratio $(\mathrm{RER})=\mathrm{VCO}_{2}$ $/ \mathrm{VO}_{2}$ and energy expenditure $(\mathrm{EE})=(3.815+1.232 \times$ $\mathrm{RER}) \times \mathrm{VO}_{2} \times 0.001(\mathrm{kcal} /[\mathrm{kg} \times \mathrm{h}])$. Due to the stress brought on by metabolic chambers, which can lead to great weight loss and even death, as we observed in our pilot study, mice from the RYGB group were divided evenly into two groups: one group was put into the chamber at 4 and 8 weeks after surgery, and the other group at 12 weeks after surgery.

\section{Glucose Metabolism}

Mice were pre-stimulated for 1 week and fasted overnight before testing at 11 weeks after surgery. The intraperitoneal glucose-tolerance test (IPGTT) was performed after 
administering glucose solution ( $1.5 \mathrm{~g} / \mathrm{kg}$ body weight) by intraperitoneal injection, while the insulin-tolerance test (ITT) was conducted with intraperitoneal injection of insulin at $0.75 \mathrm{U} / \mathrm{kg}$ body weight after 6 hours' fasting. In both experiments, blood-glucose levels were measured from tail cuts at $0,15,30,60$, and 120 minutes after injection. Glucose and insulin tolerance were assessed by calculating the area under the glucose curves (AUC).

\section{Statistical Analysis}

Statistical analysis was performed using SPSS 19.0 and Prism 6 software with ANOVA or Student's $t$-test. Data are expressed as means \pm SEM. $P<0.05$ was considered statistically differences.

\section{Results}

Mice were euthanized and underwent laparotomy at 12 weeks after surgery. Abdominal viscera and entire alimentary tracts were examined to ensure the complete exclusion of stomach and patency of anastomosis (Figure 1E).
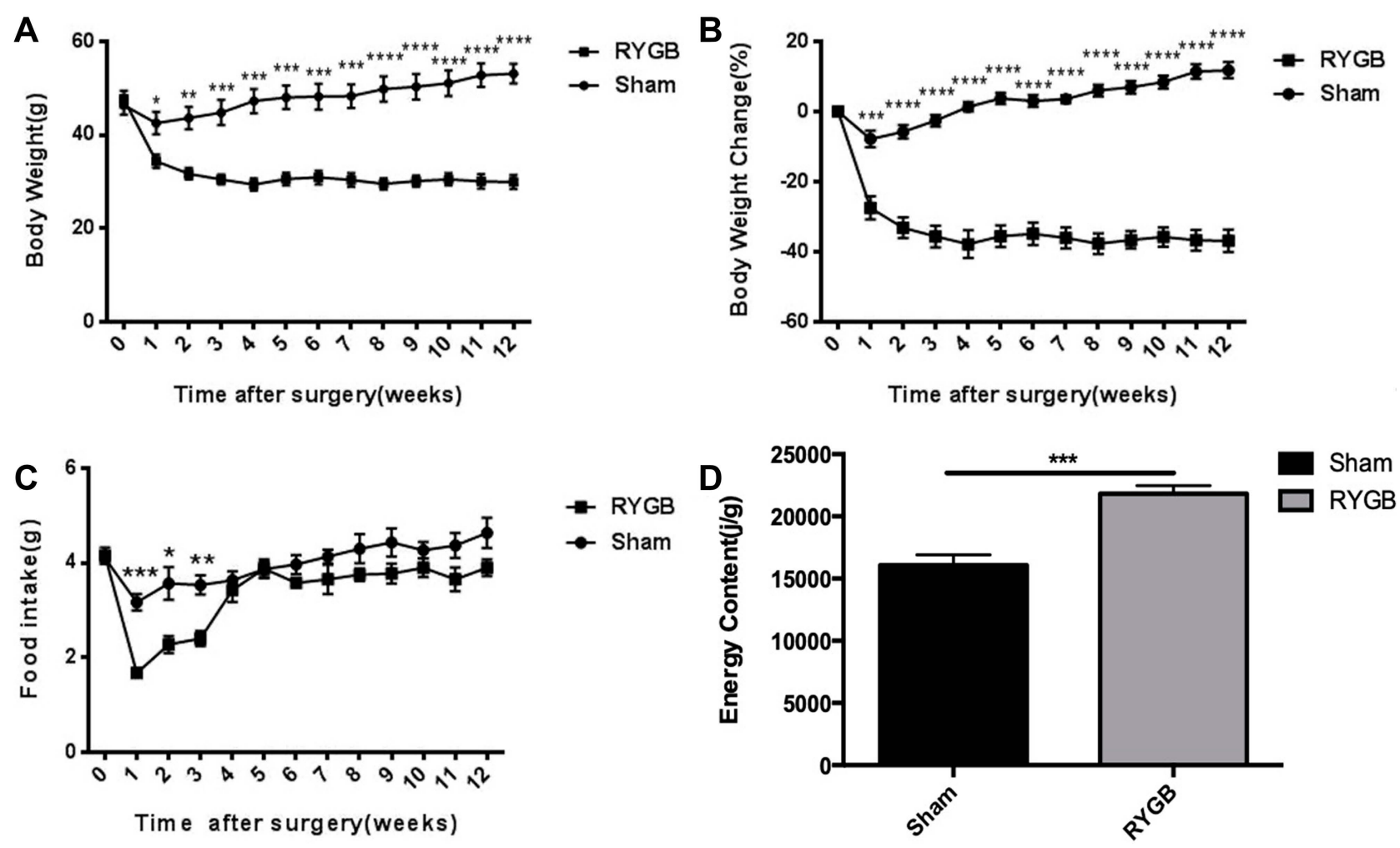

\section{RYGB Resulted in More Body-Weight Loss Without Reducing Food Intake}

The body weight of mice in the RYGB group dropped significantly in the first 2 weeks after surgery and continued to decline for 2 more weeks, until approximately $36 \% \pm 3.7 \%$ original body weight had been lost, then remained stable for the next 8 weeks. In the sham group, mice lost about $10 \%$ $\pm 1.3 \%$ body weight in the first week after surgery due to surgical stress, then started to regain weight gradually. At 4 weeks after surgery, body weight had already exceeded preoperative levels in the sham group (Figure 2A and B). Food-intake measurement demonstrated that mice in the RYGB group ate less in the first 3 weeks than the sham group. From postoperative week 4, food intake was no different between the two groups (Figure 2C).

\section{Fecal Energy Measurement Revealed That More Energy Was Lost Through Defecation after RYGB}

Feces collected at 11 weeks after surgery were weighed and measured for energy levels. There was a significantly higher

Figure 2 RYGB led to durable weight loss and increased fecal energy loss. (A) Absolute body weight significantly lower in RYGB group after surgery; (B) percentage change in body weight significantly higher in RYGB group after surgery; (C) food intake significantly reduced in RYGB group at postoperative weeks I, 2, and 3, and increased to same level as sham group at weeks 4 and thereafter; (D) fecal energy significantly higher in RYGB group at postoperative week 12 . Values are means \pm SEM. Statistical differences analyzed by one-way ANOVA followed by Student's $t$-test. $* P<0.05$ vs sham; $* * P<0.01$ vs sham; $* * * P<0.001$ vs sham; $* * * * P<0.000$ I vs sham. 
level of energy in feces from the RYGB group than the sham group (Figure 2D), indicating energy from food was lost markedly through feces in the long term after RYGB (12 weeks in mouse life span, equal to 7 years in human life span). ${ }^{12}$

\section{RYGB Reduced Fat Mass and Changed Body Composition in the Long Term}

Body-composition measurement revealed that fat-mass percentage had substantially decreased and lean-mass percentage increased in the RYGB group compared with the sham group at 4 weeks after RYGB. Although the exact weight of lean mass was lowered by RYGB (data not shown) from postoperative week 4 , the adiposity index was significantly reduced, indicating that body composition had become leaner. This alteration in body composition was maintained in the long term, as it remained unchanged at 12 weeks after RYGB (Figure 3).

EE had significantly increased at 8 weeks after surgery, and preferential substrate utilization for energy production was switched from fat to glucose at 12 weeks postoperatively. Data from the metabolic chambers showed that EE during the night was significantly higher in the RYGB group than the sham group at 8 weeks postoperatively. However, at 12 weeks after surgery, there was no difference between the groups (Figure 4A). RER values were found to be no different, significantly decreased, and significantly increased in the RYGB group compared to the sham group at 4,8 , and 12 weeks after surgery, respectively (Figure 4B), suggesting that preferential substrate utilization for energy production kept changing in the long-term postoperative course, specifically from fat at postoperative week 8 to glucose at 12 weeks after surgery.

\section{RYGB Improved Glucose Tolerance and Insulin Sensitivity in the Long Term}

Mice in the RYGB group showed significant lower levels of blood glucose than mice in the sham group on the IPGTT (Figure 5, A and B). The ITT also revealed that blood-glucose levels decreased in the RYGB group, in contrast to the sham group (Figure 5, C and D), suggesting mice in the RYGB group responded better to insulin than the sham group at 12 weeks postoperatively.

\section{Discussion}

Although different mouse models have been used for investigating the mechanism of RYGB in many studies, ${ }^{9,10,13}$ it is technically demanding to perform RYGB on DIO mice. In this study, we succeded in establishing an effective RYGB model with no mortality, and surgical techniques were introduced in detail. Bypassing the small bowel is a key component of the bariatric and metabolic benefit of RYGB, but it can also lead to malnutrition if too much small bowel is excluded. In our pilot study, the length of BP limb (measured from Treitz ligament, as normal in human surgery) and AL limb were first set to be $6 \mathrm{~cm}$ each, which led to death from malnutrition within 5 weeks. With reference to a previous study that used a $6 \mathrm{~cm}$-long AL limb and $2 \mathrm{~cm}$-long BP limb, ${ }^{13}$ we shortened the length of both to $4 \mathrm{~cm}$ each, which resulted in $36 \% \pm 3.7 \%$ average body-weight loss without malnutrition. Despite the small gastric pouch being used in the mouse model of RYGB, ${ }^{13}$ we found it would cause high mortality to create such a gastric pouch on DIO mouse from our previous attempts. Therefore, we used a modified RYGB model in which the EGJ was ligated to bypass the whole stomach, leaving the distal part of esophagus for the anastomosis to the AL limb. ${ }^{9}$ The outcome of weight loss
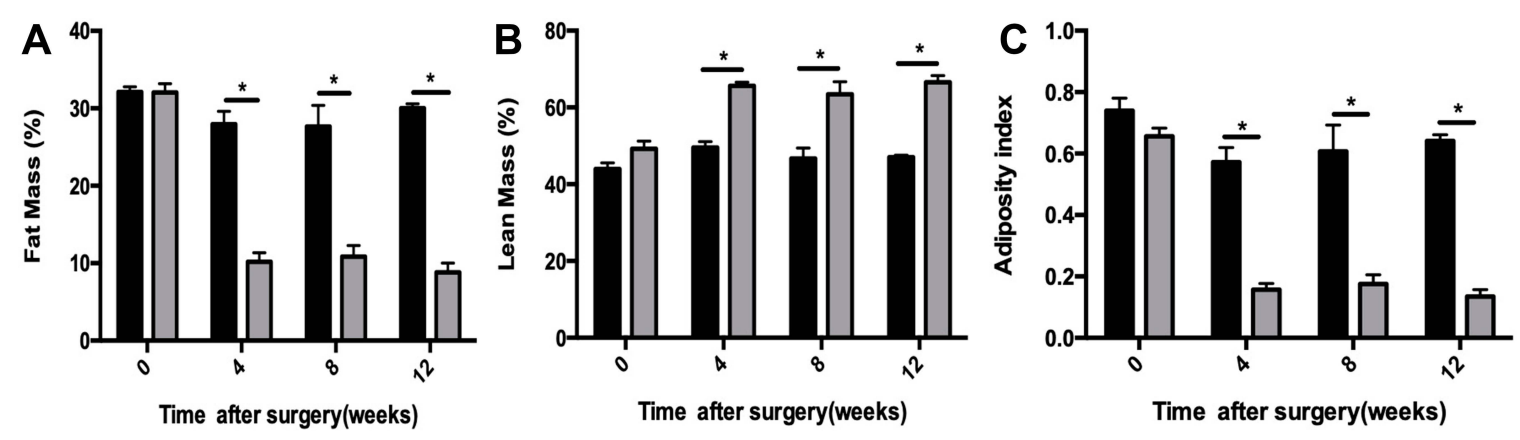

Figure 3 RYGB reduced fat mass and adiposity index. (A) Percentage of fat mass calculated as ratio of fat mass to body weight; (B) percentage of lean mass calculated as ratio of lean mass to body weight; (C) adiposity index calculated as ratio of fat mass to lean mass. Statistical differences analyzed by one-way ANOVA followed by Student's $t$-test. $* P<0.05$ vs sham. 


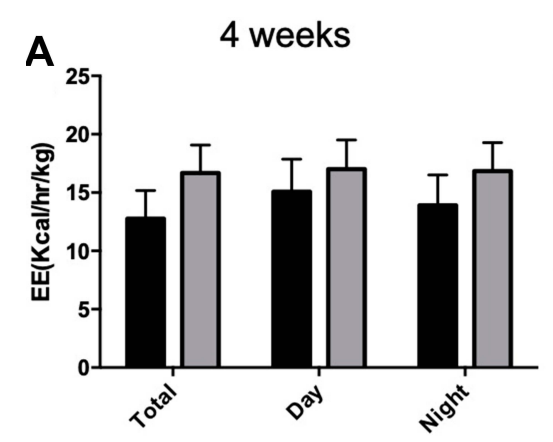

8 weeks
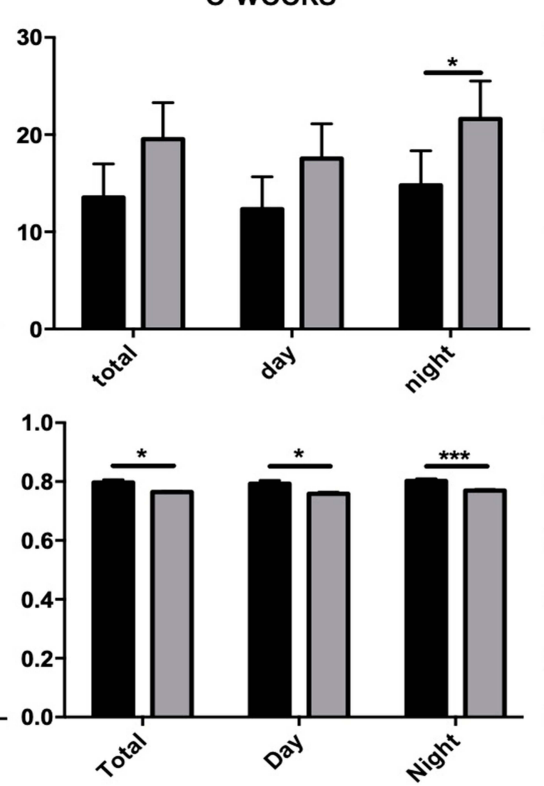

12 weeks
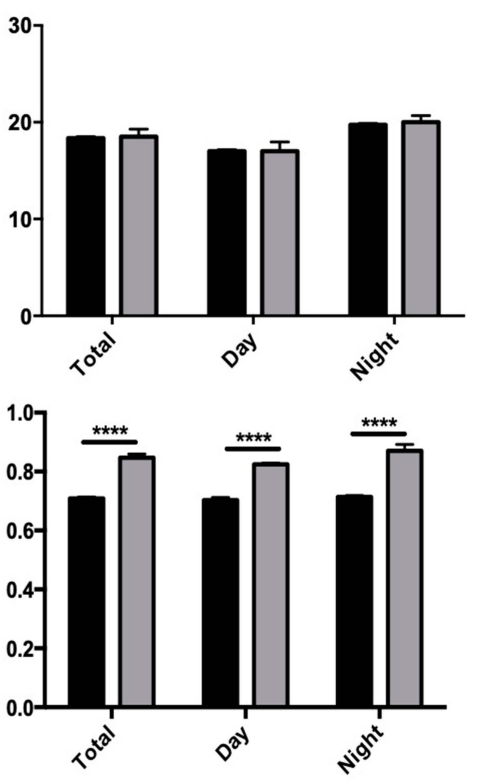

Figure 4 RYGB had altered energy expenditure by postoperative week 8 and respiratory exchange ratio by 4,8 , and 12 weeks after surgery. (A) Energy expenditure per day normalized to total body weight; (B) respiratory exchange radio. Statistical differences analyzed by one-way ANOVA followed by Student's $t$-test. $* P<0.05$ vs sham; $* * * P<0.001$ vs sham; $* * * * P<0.0001$ vs sham.

and glucose metabolism in this study was consistent with reports using a gastric pouch, suggesting the effects of RYGB could be replicated in the presented mouse model. Tips for technical improvements presented in this report could be helpful to ensure the safety and efficiency of this surgical procedure.

Similarly to other reports, ${ }^{12-15}$ body weight and food intake both declined within the first 2 weeks in the sham group, then started to increase thereafter, indicating the impact of surgical stress lasted for about 2 weeks. Mice in the RYGB group, however, continued to lose weight until 5 weeks after surgery. At 12 weeks after surgery, unlike the mice in the sham group, which regained all the lost weight and became $16 \% \pm 2 \%$ heavier, the body weight of mice in the RYGB group remained low level, despite the food intake being increased to the same as the sham group from postoperative week 4. These findings demonstrated that RYGB is effective on long-term bodyweight control, which is consistent with other studies. ${ }^{12-15}$ Although some investigations have found that food intake increased significantly late after RYGB, ${ }^{13,16}$ there was no difference between the RYGB group and sham group from postoperative week 4 , which might partially be caused by the fact that the entire stomach was excluded in our model, instead of leaving a gastric pouch.

Excessive fat accumulation was the key factor in the development of insulin resistance and metabolic syndrome, and thus reductions in fat mass would ameliorate metabolic disorders. ${ }^{17-19}$ Clinical studies have proved that RYGB can lead to higher remission rates of T2DM than vertical sleeve gastrectomy or long-term medical treatment. ${ }^{3,20}$ In this study, body composition was found to be changed by RYGB, demonstrated by reduced fat mass, reduced adiposity index, and increased percentage of lean mass over body weight at all time points in postoperative weeks 4,8 , and 12 . Therefore, it could be concluded that fat-mass reduction is the major contributor in body-weight loss after RYGB and this effect remains unchanged in the long term. As a result, insulin sensitivity was significantly improved, as reflected by the ITT. In addition, prompt insulin response to glucose uptake was enhanced by RYGB, supported by the finding of IPGTTs that the time point of blood-glucose peak level was 15 minutes in RYGB group, which was earlier than that in sham group. Notably, these measurements were conducted at 12 weeks after surgery, proving that the bariatric and metabolic effects of RYGB were maintained in the long term after surgery.

Excessive energy transformed into lipids was considered the cause of obesity, ${ }^{21,22}$ and thus the balance of energy input and output is the key factor that regulates body weight. At 4 weeks after surgery, EE was not different in sham group, despite body weight being significantly reduced in the RYGB group. However, food intake was remarkably less in the RYGB group within the first 3 weeks after surgery compared 

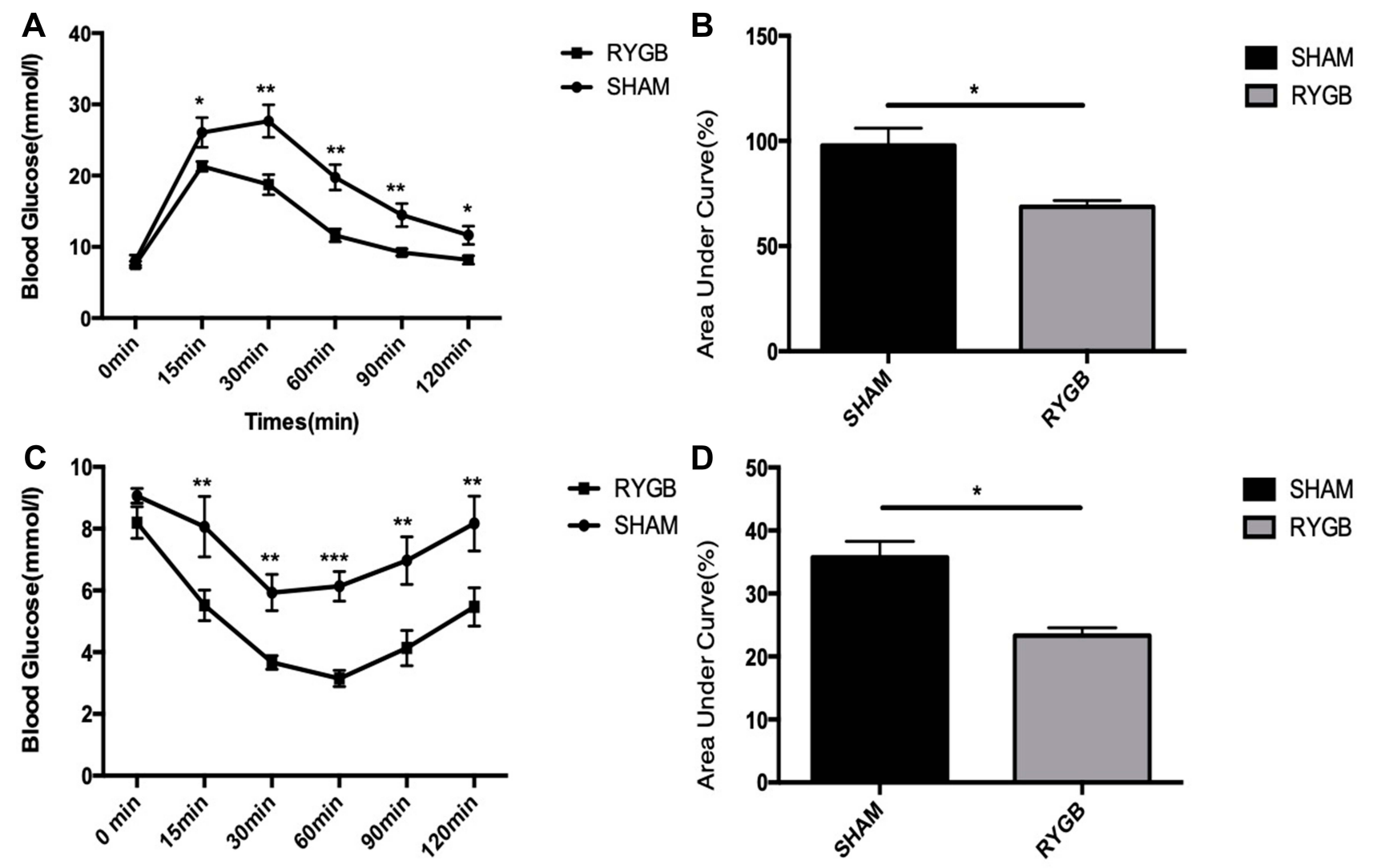

Figure 5 RYGB improved glucose metabolism. (A) Glucose excursion curves after IPGTT between sham and RYGB at postoperative week I2; (B) AUC for IPGTT between sham and RYGB at postoperative week I2; (C) glucose excursion curves after ITT between sham and RYGB at postoperative week I2; (D) AUC for ITT between sham and RYGB at postoperative week 12. Statistical differences analyzed by one-way ANOVA followed by Student's $t$-test. $* P<0.05$ vs sham; $* * P<0.0$ I vs sham; $* * * P<0.00$ I vs sham.

to the sham group, which might have been the main contributor to body-weight loss during this period. After postoperative week 4, food intake in RYGB group increased to the same as the sham group, but body weight remained low. Metabolic chambers revealed that EE had become significantly higher in the RYGB group at postoperative week 8 during the dark cycle, suggesting increased energy output might play an important role in maintaining low body weight in this period. EE was increased during the light cycle at 9 weeks after RYGB, according to Hao et al's report. ${ }^{13}$ In our study, though there was a trend of EE enhancement in the RYGB group, the difference was not significant between the groups, which might have resulted from the small sample. At 12 weeks after surgery, however, EE in the RYGB group had dropped back to the same as the sham group, indicating the augmentation effect of RYGB on EE was not durable in the long term. Taking the same food intake into consideration, some other factor might have contributed to the maintenance of low body weight in the RYGB group. The alimentary tract is realigned after RYGB, which alters the digestion and absorption of nutrients. ${ }^{23,24}$ A study on rats found that the gross energy content of feces was significantly greater in the RYGB group than the sham group. ${ }^{25}$ Fecal energy analysis in our study proved that there was more energy contained infeces from mice that had undergone RYGB than sham surgery, suggesting RYGB led to more energy loss through defecation. Therefore, increased energy loss through defecation might contribute to maintaining low body weight in the long term after surgery.

The RER represents preferential substrate utilization for producing mitochondrial ATP, and is usually used for determining the ratio of fat and carbohydrates as energy resource. ${ }^{26}$ At week 4 after surgery, RER was not different between the two groups, meaning the preferential resource of energy production had not been changed by RYGB yet. At postoperative week 8, RER had decreased, suggesting there was more fat utilized to produce energy by mice in the RYGB group than the sham group. At week 12 after surgery, the RYGB group showed increased RER, suggesting that mice used more glucose for energy production. Therefore, although the effect of raising EE by RYGB was diminished in the long term, glucose utilization was 
enhanced, which could be beneficial for maintaining normal blood-glucose levels. These findings might help to explain why some patients retain normal glucose metabolism despite body-weight regain many years after RYGB.

\section{Conclusion}

This study revealed the long-term energy-metabolism profile after RYGB with regard to EE, preferential substrate utilization, and fecal energy loss, implicating multiple mechanisms might be involved in the long-term effects of RYGB. These findings will provide new insights for the future study of metabolic surgery and obesity.

\section{Abbreviations}

RYGB, Roux-en-Y gastric bypass; IPGTT, intraperitoneal glucose-tolerance test; ITT, insulin-tolerance test; RER, respiratory exchange ratio; EGJ, esophagogastric junction; DIO, diet-induced obesity; BP, biliopancreatic; AL, alimentary.

\section{Data Sharing Statement}

The data sets used and/or analyzed during the current study are available from the corresponding author on reasonable request.

\section{Ethics and Consent Statement}

Under the Guide for Care and Use of Laboratory Animals issued by the Ministry of Science and Technology of China in 2006, the this study was approved by Animal Ethical and Welfare Committee of the Second Xiangya Hospital of Central South University (approval 202335).

\section{Acknowledgment}

This study was supported by the National Natural Science Foundation of China (81670481).

\section{Author Contributions}

All authors made substantial contributions to conception and design, acquisition of data, or analysis and interpretation of data, took part in drafting the article or revising it critically for important intellectual content, gave final approval of the version to be published, and agree to be accountable for all aspects of the work.

\section{Disclosure}

The authors have no conflicts of interest to disclose.

\section{References}

1. Yska JP, van Roon EN, de Boer A, et al. Remission of type 2 diabetes mellitus in patients after different types of bariatric surgery: a population-based cohort study in the United Kingdom. JAMA Surg. 2015;150(12):1126-1133. doi:10.1001/jamasurg.2015.2398

2. Buchwald H, Estok R, Fahrbach K, et al. Weight and type 2 diabetes after bariatric surgery: systematic review and meta-analysis. $\mathrm{Am}$ J Med. 2009;122(3):248-256.e5. doi:10.1016/j.amjmed.2008.09.041

3. Schauer PR, Bhatt DL, Kewan JP, et al. Bariatric surgery versus intensive medical therapy for diabetes - 5-year outcomes. $N$ Engl J Med. 2017;376(7):641-651. doi:10.1056/NEJMoa1600869

4. Brito JP, Montori VM, Davis AM. Metabolic surgery in the treatment algorithm for type 2 diabetes: a joint statement by international diabetes organizations. JAMA. 2017;317(6):635-636. doi:10.1001/ jama.2016.20563

5. Flum DR, Devlin A, Wright AS, et al. Development of a porcine Roux-en-Y gastric bypass survival model for the study of post-surgical physiology. Obes Surg. 2007;17(10):1332-1339. doi:10.1007/s11695-007-9237-5

6. Elward A, Fahmy M, Abu-Seida AM. Greater curvature as a gastric pouch for sleeve gastrectomy: a novel bariatric procedure. Feasibility study in a canine model. Surg Obes Relat Dis. 2018;14 (12):1814-1820. doi:10.1016/j.soard.2018.08.018

7. Lopez PP, Nicholson SE, Burkhardt GE, Johnson RA, Johnson FK. Development of a sleeve gastrectomy weight loss model in obese Zucker rats. J Surg Res. 2009;157(2):243-250. doi:10.1016/j. jss.2008.10.025

8. Liu W, Zassoko R, Mele T, et al. Establishment of duodenojejunal bypass surgery in mice: a model designed for diabetic research. Microsurgery. 2008;28(3):197-202. doi:10.1002/micr.20454

9. Yin DP, Gao Q, Ma LL, et al. Assessment of different bariatric surgeries in the treatment of obesity and insulin resistance in mice. Ann Surg. 2011;254(1):73-82. doi:10.1097/SLA.0b013e3182197035

10. Bruinsma BG, Uygun K, Yarmush ML, Saeidi N. Surgical models of Roux-en-Y gastric bypass surgery and sleeve gastrectomy in rats and mice. Nat Protoc. 2015;10(3):495-507. doi:10.1038/nprot.2015.027

11. Hariri N, Thibault L. High-fat diet-induced obesity in animal models. Nutr Res Rev. 2010;23(2):270-299. doi:10.1017/S0954422410000168

12. Hao Z, Townsend RL, Mumphrey MB, Morrison CD, Münzberg H, Berthoud HR. RYGB produces more sustained body weight loss and improvement of glycemic control compared with VSG in the diet-induced obese mouse model. Obes Surg. 2017;27(9):2424-2433. doi:10.1007/s11695-017-2660-3

13. Hao Z, Zhao Z, Berthoud HR, Ye J. Development and verification of a mouse model for Roux-en-Y gastric bypass surgery with a small gastric pouch. PLoS One. 2013;8(1):e52922. doi:10.1371/journal. pone.0052922

14. Seyfried F, Lannoo M, Gsell W, et al. Roux-en-Y gastric bypass in mice-surgical technique and characterisation. Obes Surg. 2012;22 (7):1117-1125. doi:10.1007/s11695-012-0661-9

15. Hao Z, Mumphrey MB, Townsend RL, et al. Body composition, food intake, and energy expenditure in a murine model of roux-en-y gastric bypass surgery. Obes Surg. 2016;26(9):2173-2182. doi:10.1007/ s11695-016-2062-y

16. Barkholt P, Pedersen PJ, Hay-Schmidt A, Jelsing J, Hansen HH, Vrang N. Alterations in hypothalamic gene expression following Roux-en-Y gastric bypass. Mol Metab. 2016;5(4):296-304. doi:10.1016/j.molmet.2016. 01.006

17. Hammarstedt A, Gogg S, Hedjazifar S, Nerstedt A, Smith U. Impaired adipogenesis and dysfunctional adipose tissue in human hypertrophic obesity. Physiol Rev. 2018;98(4):1911-1941. doi:10. 1152/physrev.00034.2017

18. Reilly SM, Saltiel AR. Adapting to obesity with adipose tissue inflammation. Nat Rev Endocrinol. 2017;13(11):633-643. doi:10. 1038/nrendo.2017.90 
19. Caprio S, Perry R, Kursawe R. Adolescent obesity and insulin resistance: roles of ectopic fat accumulation and adipose inflammation. Gastroenterology. 2017;152(7):1638-1646. doi:10.1053/j.gastro. 2016.12.051

20. Jakobsen GS, Småstuen MC, Sandbu R, et al. Association of bariatric surgery vs medical obesity treatment with long-term medical complications and obesity-related comorbidities. JAMA. 2018;319 (3):291-301. doi:10.1001/jama.2017.21055

21. Hajer GR, van Haeften TW, Visseren FL. Adipose tissue dysfunction in obesity, diabetes, and vascular diseases. Eur Heart J. 2008;29 (24):2959-2971. doi:10.1093/eurheartj/ehn387

22. González MP, Mártinez MA, Hu FB, et al. Obesity. Nat Rev Dis Primers. 2017;15(3):17034. doi:10.1038/nrdp.2017.34

23. Carswell KA, Vincent RP, Belgaumkar AP, et al. The effect of bariatric surgery on intestinal absorption and transit time. Obes Surg. 2014;24(5):796-805. doi:10.1007/s11695-013-1166-x
24. Bojsen-Moller KN, Jacobsen SH, Dirksen C, et al. Accelerated protein digestion and amino acid absorption after Roux-en-Y gastric bypass. Am J Clin Nutr. 2015;102(3):600-607. doi:10.3945/ ajcn. 115.109298

25. Shin AC, Zheng H, Townsend RL, Patterson LM, Holmes GM, Berthoud HR. Longitudinal assessment of food intake, fecal energy loss, and energy expenditure after Roux-en-Y gastric bypass surgery in high-fat-fed obese rats. Obes Surg. 2013;23(4):531-540. doi:10.1007/s11695-012-0846-2

26. Lu Z, Wei X, Sun F, et al. Non-insulin determinant pathways maintain glucose homeostasis upon metabolic surgery. Cell Discov. 2018;4 (1):58. doi:10.1038/s41421-018-0062-x

Diabetes, Metabolic Syndrome and Obesity: Targets and Therapy

\section{Publish your work in this journal}

Diabetes, Metabolic Syndrome and Obesity: Targets and Therapy is an international, peer-reviewed open-access journal committed to the rapid publication of the latest laboratory and clinical findings in the fields of diabetes, metabolic syndrome and obesity research. Original research, review, case reports, hypothesis formation, expert opinion and commentaries are all considered for publication. The manuscript management system is completely online and includes a very quick and fair peer-review system, which is all easy to use. Visit http://www.dovepress.com/testimonials.php to read real quotes from published authors. 